(1)

Noberentiver Journal of Nonlinear Mathematical Physics

ISSN (Online): 1776-0852 ISSN (Print): 1402-9251

Journal Home Page: https://www.atlantis-press.com/journals/jnmp

\title{
A class of third-order nonlinear evolution equations admitting invariant subspaces and associated reductions
}

Yujian Ye, Wen-Xiu Ma, Shoufeng Shen, Danda Zhang

To cite this article: Yujian Ye, Wen-Xiu Ma, Shoufeng Shen, Danda Zhang (2014) A class of third-order nonlinear evolution equations admitting invariant subspaces and associated reductions, Journal of Nonlinear Mathematical Physics 21:1, 132-148, DOI: https://doi.org/10.1080/14029251.2014.894726

To link to this article: https://doi.org/10.1080/14029251.2014.894726

Published online: 04 January 2021 


\title{
A class of third-order nonlinear evolution equations admitting invariant subspaces and associated reductions
}

\author{
Yujian $\mathrm{Ye}^{1}$, Wen-Xiu Ma ${ }^{2}, \quad$ Shoufeng Shen ${ }^{3, *}$, Danda Zhang ${ }^{3}$ \\ ${ }^{1}$ School of Management, Hangzhou Dianzi University, \\ Hangzhou 310018, P.R. China \\ 2 Department of Mathematics and Statistics, University of South Florida, \\ Tampa, FL 33620-5700, USA \\ ${ }^{3}$ Department of Applied Mathematics, Zhejiang University of Technology, \\ Hangzhou 310023, P.R. China
}

manageyyj@163.com,mawx@cas.usf.edu,mathssf@zjut.edu.cn,mathddz@163.com

Received 24 October 2013

Accepted 23 December 2013

\begin{abstract}
With the aid of symbolic computation by Maple, a class of third-order nonlinear evolution equations admitting invariant subspaces generated by solutions of linear ordinary differential equations of order less than seven is analyzed. The presented equations are either solved exactly or reduced to finite-dimensional dynamical systems. A number of concrete examples admitting invariant subspaces generated by power, trigonometric and exponential functions are computed to illustrate the resulting theory.
\end{abstract}

Keywords: evolution equation; invariant subspace; separation of variables; reduction; dynamical system.

2000 Mathematics Subject Classification: 35G20

\section{Introduction}

In this paper, we analyze a class of third-order nonlinear evolution equations (NLEEs) with the following form

$$
u_{t}=f(u) u_{x x x}+g(u) u_{x x} u_{x}+h(u) u_{x}^{3}+q_{1}(x) q_{2}(u)+q_{3}(u)
$$

which admit invariant subspaces defined by linear ordinary differential equations (ODEs). For the sake of convenience, we set

$$
Q(x, u)=q_{1}(x) q_{2}(u)+q_{3}(u) .
$$

The class of equations (1.1) includes some important equations of mathematical physics. For example, if we take

$$
f(u)=-\frac{1}{2} u^{-\frac{3}{2}}, g(u)=\frac{9}{4} u^{-\frac{5}{2}}, h(u)=-\frac{15}{8} u^{-\frac{7}{2}}, Q(x, u)=0
$$

$\overline{\text { *Corresponding author. }}$ 
Eq.(1.1) becomes the famous Harry Dym equation:

$$
u_{t}=\left(\frac{1}{\sqrt{u}}\right)_{x x x},
$$

whose extended hierarchy of soliton flows, including the Harry Dym hierarchy with self-consistent sources and the k-constrained Harry Dym hierarchy, was presented in [11].

Many researchers engaged in the development of the invariant subspace method, a very effective approach for constructing various types of exact solutions [2,4-7]. Kamran, Milson and Olver have provided the rigorous foundations for the invariant subspace method and the fundamental problem of maximal dimension of invariant subspaces is established first by Galaktionov and Svirshchevskii for the scalar case. Various invariant subspaces to NLEEs of the form

$$
u_{t}=f\left(u, u_{x}\right) u_{x x}+g\left(u, u_{x}\right)
$$

with certain functions $f$ and $g$ have been obtained, which yield a number of interesting exact solutions of the corresponding equations. For example, an exact solution of the quasi-linear heat equation

$$
u_{t}=\left(u^{-\frac{4}{3}} u_{x}\right)_{x}-u^{-\frac{1}{3}}
$$

can be constructed based on the 5-dimensional trigonometric invariant subspace

$$
\mathscr{L}\{1, \sin (2 / \sqrt{3} x), \cos (2 / \sqrt{3} x), \sin (4 / \sqrt{3} x), \cos (4 / \sqrt{3} x)\} .
$$

The fast diffusion equation

$$
u_{t}=\left(u^{-\frac{3}{2}} u_{x}\right)_{x}
$$

has an exact solution which belongs to the 4-dimensional polynomial invariant subspace

$$
\mathscr{L}\left\{1, x, x^{2}, x^{3}\right\} .
$$

It is also shown that multi-soliton solutions of integrable equations derived by Hirota's bilinear method belong to invariant subspaces of exponential functions in the sense of change of variables. So many different types of exact solutions to NLEEs can be obtained through the invariant subspace method.

The invariant subspace method can also be used to construct exact solutions to systems of NLEEs (see, e.g, [8]). In Ref. [16], authors have provided a classification to systems with twocomponent nonlinear diffusion equations based on the invariant subspaces defined by linear ODEs. In Refs. [17, 18, 21], authors have generalized the estimation of maximal dimension of invariant subspaces to the vector operators. Noting that a solution to an $n$ th-order linear ODE may not satisfy another linear ODE of order less than $n$, the invariant subspace method has been refined for the unity and diversity of invariant subspaces and exact solutions in [9]. The refined invariant subspace method has been used to classify a class of systems of nonlinear dispersive evolution equations and determine their invariant subspaces and exact solutions [10]. In addition, the invariant subspace method has also been modified to deal with fractional order differential equations in Ref. [3]. It allows one to reduce a fractional order partial differential equation to a fractional order dynamical system. 
Let us give a brief account of the invariant subspace method. We consider a $k$ th-order NLEE of the form

$$
u_{t}=F\left(x, u, u_{x}, u_{x x}, \cdots, u_{k x}\right) \equiv F[u],
$$

where $F[u]$ is a given sufficiently smooth function of the indicated variables. Let $\left\{f_{i}(x), i=1, \cdots, n\right\}$ be a finite set of $n(\geq 1)$ linearly independent functions, and $W_{n}$ denote their linear span

$$
W_{n}=\mathscr{L}\left\{f_{1}(x), \cdots, f_{n}(x)\right\} \equiv\left\{c_{1} f_{1}(x)+\cdots+c_{n} f_{n}(x) \mid \forall c_{i} \in \mathbb{R}\right\} .
$$

The given operator $F$ (or the given evolution equation (1.3)) admits an invariant space $W_{n}$, i.e., the subspace $W_{n}$ is said an invariant subspace of the given operator $F$, if the invariance condition

$$
F\left[W_{n}\right] \subseteq W_{n}
$$

holds. Concretely speaking, this invariance condition means

$$
F\left[\sum_{i=1}^{n} c_{i} f_{i}(x)\right]=\sum_{i=1}^{n} \Psi_{i}\left(c_{1}, \cdots, c_{n}\right) f_{i}(x) \text { for any }\left(c_{1}, \cdots, c_{n}\right) \in \mathbb{R}^{n},
$$

where the expansion coefficients $\left\{\Psi_{i}\right\}$ of $F[u] \in W_{n}$ in the basis $\left\{f_{i}\right\}$ are a set of functions of $c_{1}, c_{2}, \cdots, c_{n}$. Now it is obvious that if a linear subspace $W_{n}$ is invariant under $F$, then Eq. (1.3) has solutions of the form

$$
u(x, t)=\sum_{i=1}^{n} c_{i}(t) f_{i}(x)
$$

where the coefficients $\left\{c_{i}(t)\right\}$ satisfy an $n$-dimensional dynamical system

$$
c_{i}^{\prime}(t)=\Psi_{i}\left(c_{1}(t), \cdots, c_{n}(t)\right), 1 \leq i \leq n .
$$

Assume that an invariant subspace $W_{n}$ is determined by the space of solutions of an $n$ th-order linear ODE

$$
L[y] \equiv y^{(n)}+a_{1}(x) y^{(n-1)}+\cdots+a_{n-1}(x) y^{\prime}+a_{n}(x) y=0 .
$$

If the operator $F[u]$ admits the invariant subspace defined by the linear ODE (1.9), then the invariance condition with respect to $F$ becomes

$$
\left.L[F[u]]\right|_{[H]} \equiv 0,
$$

where $[H]$ denotes the equation $L[u]=0$ and its differential consequences with respect to $x$. The invariance condition (1.10) implies that the invariant subspace method is closely related to the conditional Lie-Bäcklund symmetry method and the differential constraint method $[1,6,15,20]$.

It has been known that the estimate of the dimension of invariant subspaces for differential operators plays an important role in the corresponding theory. Once the maximal dimension of invariant subspaces is identified, we can determine a complete classification of invariant subspaces for the equations under consideration. For the scalar case, the maximal dimension of invariant subspaces is not greater than $2 k+1$, where $k$ is the differential order of the operator $F[u]$ with respect to $x$. This makes it possible to search for a complete list of invariant subspaces. Consequently, we only need 
to consider invariant subspaces determined by linear ODEs (1.9) of order less than seven for Eq. (1.1).

The remainder of this paper is organized as follows. In section 2, equations of the form (1.1) admitting invariant subspaces generated by Eq.(1.9) are obtained by means of symbolic computation (Maple). Reductions and exact solutions of some concrete examples are used to illustrate the method in section 3. The last section is devoted to conclusions and discussions.

\section{Symbolic computation for invariant subspaces}

We first consider the case of $W_{2}$ generated by

$$
L[y] \equiv y^{\prime \prime}+a_{1}(x) y^{\prime}+a_{2}(x) y=0,
$$

where $a_{1}$ and $a_{2}$ are assumed to be real elementary functions. A direct computation by using the symbolic computation software, Maple, from the invariance condition (1.10) yields

$$
\begin{aligned}
\left.L[F[u]]\right|_{[H]}= & h^{\prime \prime} u_{x}^{5}-\left(g^{\prime \prime}+6 h^{\prime}\right) a_{1} u_{x}^{4}+\left[\left(4 g^{\prime}+f^{\prime \prime}+6 h\right) a_{1}^{2}-\left(2 h+f^{\prime \prime}+2 g^{\prime}+g^{\prime \prime} u+7 h^{\prime} u\right) a_{2}\right. \\
& \left.-\left(f^{\prime \prime}+3 h+2 g^{\prime}\right) a_{1}^{\prime}\right] u_{x}^{3}+\left\{-2\left(f^{\prime}+g\right) a_{1}^{3}+\left[\left(f^{\prime \prime}+7 g^{\prime}+12 h\right) u+3 g+4 f^{\prime}\right] a_{1} a_{2}\right. \\
& \left.-\left[\left(3 h+2 g^{\prime}+f^{\prime \prime}\right) u+4 f^{\prime}+2 g\right] a_{2}^{\prime}+\left(5 g+6 f^{\prime}\right) a_{1} a_{1}^{\prime}+Q_{u u}-\left(2 f^{\prime}+g\right) a_{1}^{\prime \prime}\right\} u_{x}^{2} \\
& +\left[-\left(3 f^{\prime}+4 g\right) a_{1}^{2} a_{2} u-3\left(a_{1} a_{1}^{\prime}-a_{1}^{\prime \prime}\right) f a_{1}+\left(3 g u+2 f^{\prime} u+3 f\right) a_{1} a_{2}^{\prime}\right. \\
& +3\left(g+2 h u+f^{\prime}+g^{\prime} u\right) u a_{2}^{2}+\left(5 g u+5 f^{\prime} u+3 f\right) a_{1}^{\prime} a_{2}-\left(2 f^{\prime} u+g u+3 f\right) a_{2}^{\prime \prime} \\
& \left.+3 a_{1}^{\prime 2} f+2 Q_{x u}-f a_{1}^{\prime \prime \prime}\right] u_{x}-\left[\left(2 g+f^{\prime}\right) u^{2} a_{2}^{2}+3 f a_{1}^{\prime} a_{2} u-Q_{x}\right] a_{1} \\
& +\left[\left(3 g+f^{\prime}\right) a_{2}^{\prime} u^{2}+\left(3 f a_{2}^{\prime}+3 f a_{1}^{\prime \prime}-Q_{u}\right) u+Q\right] a_{2}-f a_{2}^{\prime \prime \prime} u+3 f a_{1}^{\prime} a_{2}^{\prime} u+Q_{x x} \\
= & 0,
\end{aligned}
$$

where the prime and the subscript denote the derivative and the partial derivative with respect to the indicated variables respectively. To vanish all the coefficients of the above equation, we obtain the following overdetermined system,

$$
\begin{aligned}
& h^{\prime \prime}=0, \\
& \left(g^{\prime \prime}+6 h^{\prime}\right) a_{1}=0, \\
& \left(4 g^{\prime}+f^{\prime \prime}+6 h\right) a_{1}^{2}-\left(2 h+f^{\prime \prime}+2 g^{\prime}+g^{\prime \prime} u+7 h^{\prime} u\right) a_{2}-\left(f^{\prime \prime}+3 h+2 g^{\prime}\right) a_{1}^{\prime}=0, \\
& {\left[\left(f^{\prime \prime}+7 g^{\prime}+12 h\right) u+3 g+4 f^{\prime}\right] a_{1} a_{2}-2\left(f^{\prime}+g\right) a_{1}^{3}-\left[\left(3 h+2 g^{\prime}+f^{\prime \prime}\right) u+4 f^{\prime}+2 g\right] a_{2}^{\prime}} \\
& \quad+\left(5 g+6 f^{\prime}\right) a_{1} a_{1}^{\prime}+Q_{u u}-\left(2 f^{\prime}+g\right) a_{1}^{\prime \prime}=0, \\
& \left(3 g u+2 f^{\prime} u+3 f\right) a_{1} a_{2}^{\prime}+3\left(g+2 h u+f^{\prime}+g^{\prime} u\right) u a_{2}^{2}+\left(5 g u+5 f^{\prime} u+3 f\right) a_{1}^{\prime} a_{2} \\
& \quad-\left(2 f^{\prime} u+g u+3 f\right) a_{2}^{\prime \prime}+3 a_{1}^{\prime 2} f-\left(3 f^{\prime}+4 g\right) a_{1}^{2} a_{2} u-3\left(a_{1} a_{1}^{\prime}-a_{1}^{\prime \prime}\right) f a_{1}+2 Q_{x u}-f a_{1}^{\prime \prime \prime}=0, \\
& \left.\quad\left(3 g+f^{\prime}\right) a_{2}^{\prime} u^{2}+\left(3 f a_{2}^{\prime}+3 f a_{1}^{\prime \prime}-Q_{u}\right) u+Q\right] a_{2}-\left[\left(2 g+f^{\prime}\right) u^{2} a_{2}^{2}+3 f a_{1}^{\prime} a_{2} u-Q_{x}\right] a_{1} \\
& \quad+3 f a_{1}^{\prime} a_{2}^{\prime} u-f a_{2}^{\prime \prime \prime} u+Q_{x x}=0 .
\end{aligned}
$$


From the first equation of the above system (2.2), it is apparent that $h(u)=a u+b$. And the solution can be divided into two cases $a_{1}(x)=0$ and $a_{1}(x) \neq 0$.

Case 1: $a_{1}(x)=0$.

Substituting $h(u)$ and $a_{1}(x)$ into system (2.2), then the third equation becomes

$$
-a_{2}\left(f^{\prime \prime}+g^{\prime \prime} u+2 g^{\prime}+9 a u+2 b\right)=0 .
$$

Subcase 1.1: $a_{2}=0$.

Substituting all these into Eq.(2.2), the corresponding solution can be easily obtained and listed as the first entry in Table 1 with arbitrary functions $f(u)$ and $g(u)$.

Subcase 1.2: $a_{2} \neq 0$.

From Eq. (2.3), it is apparent $f(u)=-u g(u)-\frac{3 a}{2} u^{3}-b u^{2}+c u+d$. Substituting $f(u)$ into system (2.2), comparing the forth and fifth equations in (2.2) and using the compatibility condition $Q_{\text {uиx }}=Q_{\text {хии }}$, we have

$$
\frac{15 a}{2} u^{2}\left(a_{2}^{\prime \prime}+3 a_{2}^{2}\right)-3 c\left(a_{2}^{\prime \prime}+a_{2}^{2}\right)=0 .
$$

- If $a_{2}^{\prime \prime}+3 a_{2}^{2}=a_{2}^{\prime \prime}+a_{2}^{2}=0, a_{2}$ should be zero, which leads to a contradiction.

- If $a_{2}^{\prime \prime}+3 a_{2}^{2} \neq 0, a_{2}^{\prime \prime}+a_{2}^{2} \neq 0, a=c=0$, from the forth equation, we can derive

$$
Q(x, u)=a_{2}^{\prime}\left(-g(u) u^{2}-\frac{7}{6} u^{3} b+\frac{3}{2} d u\right)+p u .
$$

Substituting $Q(x, u)$ into the last equation of Eq.(2.2), we obtain

$$
-\frac{1}{6} b\left(a_{2}^{\prime \prime \prime}+16 a_{2}^{\prime} a_{2}\right) u^{3}+\frac{1}{2} d\left(a_{2}^{\prime \prime \prime}+6 a_{2}^{\prime} a_{2}\right) u=0 .
$$

If $b=d=0$, the corresponding solution is listed as the second entry in Table 1 with arbitrary $a_{2}(x)$. If $a_{2}^{\prime \prime \prime}+16 a_{2}^{\prime} a_{2}=a_{2}^{\prime \prime \prime}+6 a_{2}^{\prime} a_{2}=0$, we have $a_{2}=s$ and the corresponding solution is listed as the third entry in Table 1 . If $a_{2}^{\prime \prime \prime}+16 a_{2}^{\prime} a_{2}=d=0, a_{2}^{\prime \prime \prime}+6 a_{2}^{\prime} a_{2} \neq 0$, consequently we can have one special choose of $a_{2}=-\frac{3}{4 x^{2}}$ with the corresponding solution listed as the forth entry in Table 1. If $a_{2}^{\prime \prime \prime}+16 a_{2}^{\prime} a_{2} \neq 0, a_{2}^{\prime \prime \prime}+6 a_{2}^{\prime} a_{2}=0$, one choose is $a_{2}=-\frac{2}{x^{2}}$, which contradicts to $a_{2}^{\prime \prime}+3 a_{2}^{2} \neq 0$.

- If $a_{2}^{\prime \prime}+a_{2}^{2}=a=0, a_{2}^{\prime \prime}+3 a_{2}^{2} \neq 0$, we obtain $a_{2}=-\frac{6}{x^{2}}$. Furthermore, we derive

$$
Q(x, u)=\frac{1}{x^{3}}\left(-12 g(u) u^{2}-14 u^{3} b+24 c u^{2}+e u\right)+p u
$$

from the forth equation. Substituting $Q(x, u)$ into the last equation of (2.2), we derive $b=d=e=0$ with the corresponding solutions listed as the fifth entry in Table 1.

- By the similar calculation, if $a_{2}^{\prime \prime}+3 a_{2}^{2}=c=0, a_{2}^{\prime \prime}+a_{2}^{2} \neq 0$, we have a special choose of $a_{2}=-\frac{2}{x^{2}}$ and the further solution listed as the sixth entry in Table 1 .

Case 2: $a_{1}(x) \neq 0$.

It is apparent that $g(u)=-3 a u^{2}+c u+d$. Consequently, the third equation can be simplified to

$$
\left(a_{1}^{2}-a_{2}-a_{1}^{\prime}\right) f^{\prime \prime}+9 a\left(a_{2}-2 a_{1}^{2}+a_{1}^{\prime}\right) u+(6 b+4 c) a_{1}^{2}-2(b+c) a_{2}-(2 c+3 b) a_{1}^{\prime}=0 .
$$

The possibility should be divided into two subcases.

Subcase 2.1: $a_{2}=a_{1}^{2}-a_{1}^{\prime}$. 
Substituting $a_{2}$ into Eq.(2.4), it arrives at

$$
-9 a a_{1}^{2} u+(4 b+2 c) a_{1}^{2}-b a_{1}^{\prime}=0,
$$

which yields $a=0, b \neq 0$ or $a=b=c=0$.

- When $b \neq 0,4 b+2 c \neq 0$, we obtain a special choose of $a_{1}=-\frac{b}{(4 b+2 c) x}$. Renaming $a_{1}=\frac{s}{x}$, $a_{2}=\frac{s^{2}+s}{x^{2}}$, from (2.2) it is easy to see that

$$
Q(x, u)=\frac{1}{x^{3}} q_{2}(u)+p u+q .
$$

Substituting it into the fifth equation, we can find the relation between $q_{2}(u)$ and $f(u)$. Furthermore, substituting $q_{2}(u)$ into Eq.(2.2), taking account of the assumption $b \neq 0$, we can single out nonzero $s=-2,-\frac{5 \pm \sqrt{13}}{4}$ with the corresponding results presented as the 7-8th entries of Table 1 .

- When $b \neq 0,4 b+2 c=0$, we derive $a_{1}=s, a_{2}=s^{2}$. Substituting these into Eq.(2.2), we can derive $b=d=0$ from the fifth equation, which contradicts with the assumption $b \neq 0$.

- When $a=b=c=0$, using the compatibility condition $Q_{u u x}=Q_{x u u}$, we obtain $d=0$ or $d \neq 0, a_{1}=\frac{1}{x}$. However if $d \neq 0, a_{1}=\frac{1}{x}$, it leads to $d=0$. When $d=0$, by similar calculation, it is interesting to solve

$$
Q(x, v)=\left(3 a_{1}^{\prime} a_{1}-a_{1}^{3}-a_{1}^{\prime \prime}\right) f(u) u+p u,
$$

with arbitrary function $a_{1}(x)$ and $f(u)$, which is represented as the 9th entry in Table 1.

Subcase 2.2: $a_{2} \neq a_{1}^{2}-a_{1}^{\prime}$.

From Eq. (2.4), we derive

$$
f(u)=\frac{\left(\frac{3}{2} a_{2}-3 a_{1}^{2}+\frac{3}{2} a_{1}^{\prime}\right) a u^{3}}{a_{2}-a_{1}^{2}+a_{1}^{\prime}}+\frac{\left[-(b+c) a_{2}+(3 b+2 c) a_{1}^{2}-\left(\frac{3}{2} b+c\right) a_{1}^{\prime}\right] u^{2}}{a_{2}-a_{1}^{2}+a_{1}^{\prime}}+\mu_{1} u+\mu_{2},
$$

which implies

$$
\begin{aligned}
& \left(\frac{3}{2} a_{2}-3 a_{1}^{2}+\frac{3}{2} a_{1}^{\prime}\right) a=\lambda_{1}\left(a_{2}-a_{1}^{2}+a_{1}^{\prime}\right), \\
& -(b+c) a_{2}+(3 b+2 c) a_{1}^{2}-\left(\frac{3}{2} b+c\right) a_{1}^{\prime}=\lambda_{2}\left(a_{2}-a_{1}^{2}+a_{1}^{\prime}\right),
\end{aligned}
$$

where $\lambda_{1}$ and $\lambda_{2}$ are two constants. Solving this system, it is easy to see that $a_{1}$ and $a_{2}$ have special chooses: $a_{1}=s_{1}, a_{2}=s_{2}$ or $a_{1}=\frac{s_{1}}{x}, a_{2}=\frac{s_{2}}{x^{2}}$. By similar calculation, we have listed these results in Table 1 in sequence.

The unknown functions in Table 1 are given as follows:

$$
\left\{\begin{array}{l}
\theta_{1}^{(1)}(x)=1, \quad \theta_{2}^{(1)}(x)=x, \quad s=0, \\
\theta_{1}^{(1)}(x)=\sin (\sqrt{s} x), \quad \theta_{2}^{(1)}(x)=\cos (\sqrt{s} x), \quad s>0, \quad \quad \theta_{2}^{(1)}(x)=\exp (-\sqrt{-s} x), \quad s<0 \\
\theta_{1}^{(1)}(x)=\exp (\sqrt{-s} x), \quad
\end{array}\right.
$$




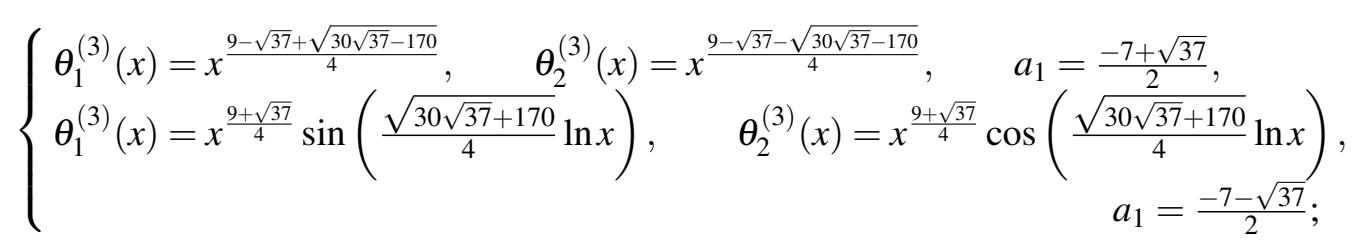

$$
\begin{aligned}
& \left\{\begin{array}{lrl}
\theta_{1}^{(4)}(x)=x^{3}, & \theta_{2}^{(4)}(x)=x^{3} \ln x, & s=-5, \\
\theta_{1}^{(4)}(x)=x^{-\frac{2 s+1}{3},} & \theta_{2}^{(4)}(x)=x^{-\frac{s-4}{3},} \quad s \neq-5 ;
\end{array}\right.
\end{aligned}
$$

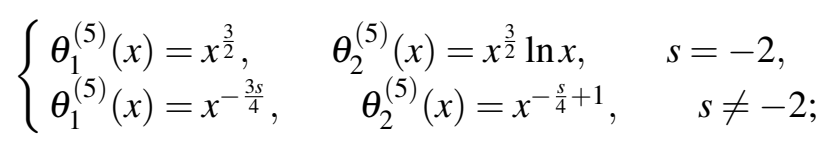

$$
\begin{aligned}
& \begin{cases}\theta_{1}^{(6)}(x)=x^{\frac{3}{2}}, & \theta_{2}^{(6)}(x)=x^{\frac{3}{2}} \ln x, \quad s=\frac{9}{4}, \\
\theta_{1}^{(6)}(x)=x^{\frac{3}{2}}, & \theta_{2}^{(6)}(x)=x^{\frac{2 s}{3}}, \quad s \neq \frac{9}{4}\end{cases} \\
& \left\{\begin{array}{l}
\theta_{1}^{(7)}(x)=x^{\frac{3}{2}}, \quad \theta_{2}^{(7)}(x)=x^{\frac{3}{2}} \ln x, \quad s=\frac{9}{4}, \\
\theta_{1}^{(7)}(x)=x^{\frac{3}{2}} \sin \left(\frac{\sqrt{4 s-9}}{2} \ln x\right), \quad \theta_{2}^{(7)}(x)=x^{\frac{3}{2}} \cos \left(\frac{\sqrt{4 s-9}}{2} \ln x\right), \quad s>\frac{9}{4} ; \\
\theta_{1}^{(7)}(x)=x^{\frac{3+\sqrt{9-4 s}}{2}}, \quad \theta_{2}^{(7)}(x)=x^{\frac{3-\sqrt{9-4 s}}{2}}, \quad s<\frac{9}{4} ;
\end{array}\right. \\
& \left\{\begin{array}{lll}
\theta_{1}^{(8)}(x)=x^{3}, & \theta_{2}^{(8)}(x)=x^{3} \ln x, & s=-5, \\
\theta_{1}^{(8)}(x)=x^{3}, & \theta_{2}^{(8)}(x)=x^{-s-2}, & s \neq-5 .
\end{array}\right.
\end{aligned}
$$

Moreover, $\theta_{1}^{(0)}(x)$ and $\theta_{2}^{(0)}(x)$ listed as the second entry satisfy the equation $y^{\prime \prime}+a_{2} y=0$, which can not be solved for arbitrary function $a_{2} \equiv a_{2}(x)$. It is the same with $\left\{\theta_{1}^{(2)}(x), \theta_{2}^{(2)}(x)\right\}$ and $\left\{\theta_{1}^{(9)}(x), \theta_{2}^{(9)}(x), \theta_{3}^{(9)}(x)\right\}$.

By similar calculation, we obtain equations with invariant subspaces $W_{n},(n=3,4,5,6)$ listed in Table 2. And the unknown functions are given as follows:

$$
\begin{gathered}
\theta_{1}^{(10)}(x)=x^{\frac{-3 \mp \sqrt{89}}{4}} \sin \left(\frac{\sqrt{ \pm 42 \sqrt{89}+398}}{4} \ln x\right) \\
\theta_{2}^{(10)}(x)=x^{\frac{-3 \mp \sqrt{89}}{4}} \cos \left(\frac{\sqrt{ \pm 42 \sqrt{89}+398}}{4} \ln x\right) \\
\left\{\begin{array}{l}
\theta_{2}^{(11)}(x)=x, \quad \theta_{3}^{(11)}(x)=x^{2}, \quad s=0, \\
\theta_{2}^{(11)}(x)=\sin (\sqrt{s} x), \quad \theta_{3}^{(11)}(x)=\cos (\sqrt{s} x), \quad s>0, \\
\theta_{2}^{(11)}(x)=\exp (\sqrt{-s} x), \quad \theta_{3}^{(11)}(x)=\exp (-\sqrt{-s} x), \quad s<0 .
\end{array}\right.
\end{gathered}
$$

For $W_{7}$, we find that the determining system is inconsistent so that there exists no invariant subspace generated by linear ODEs (1.9) for Eq.(1.1). 


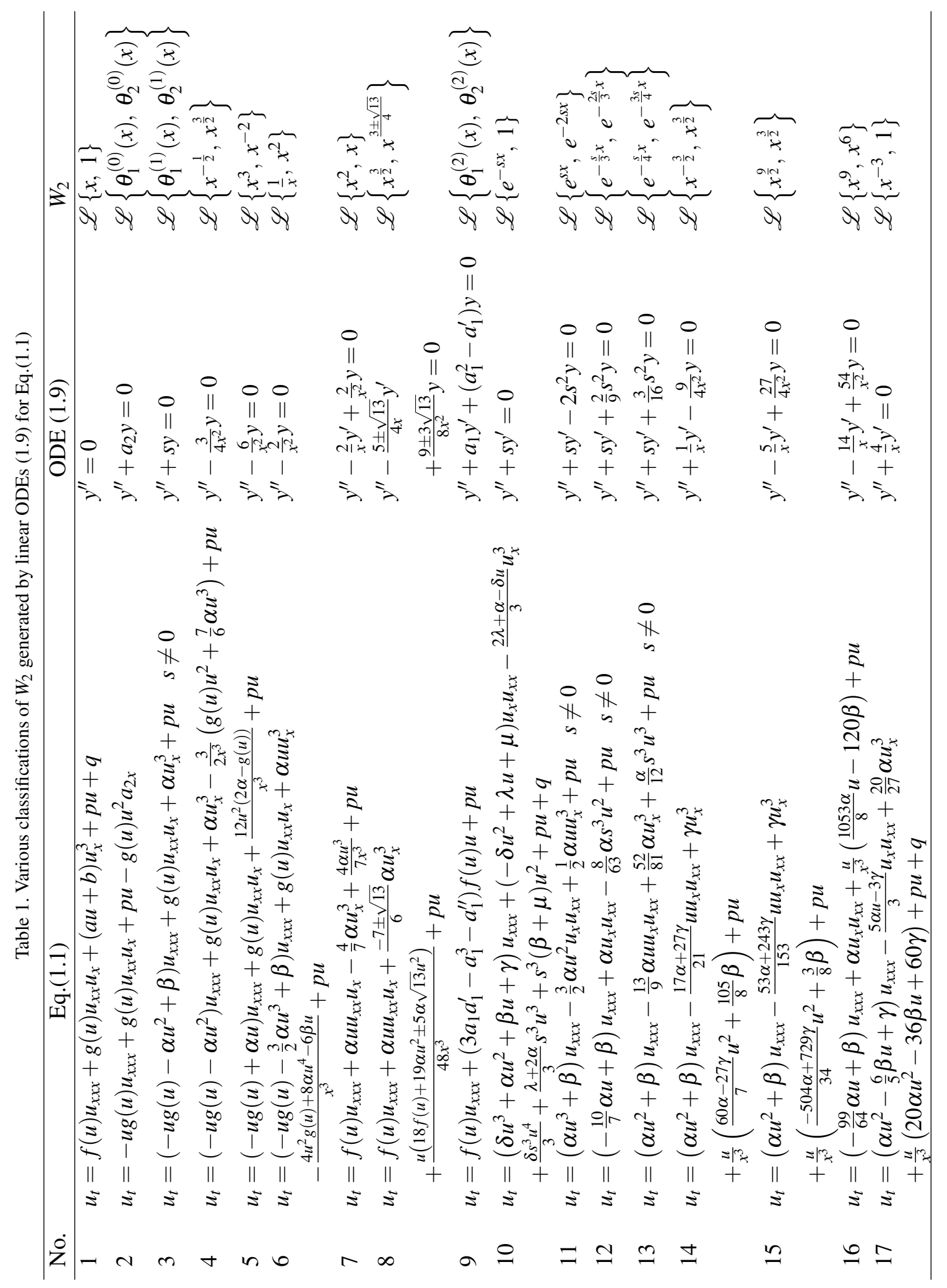




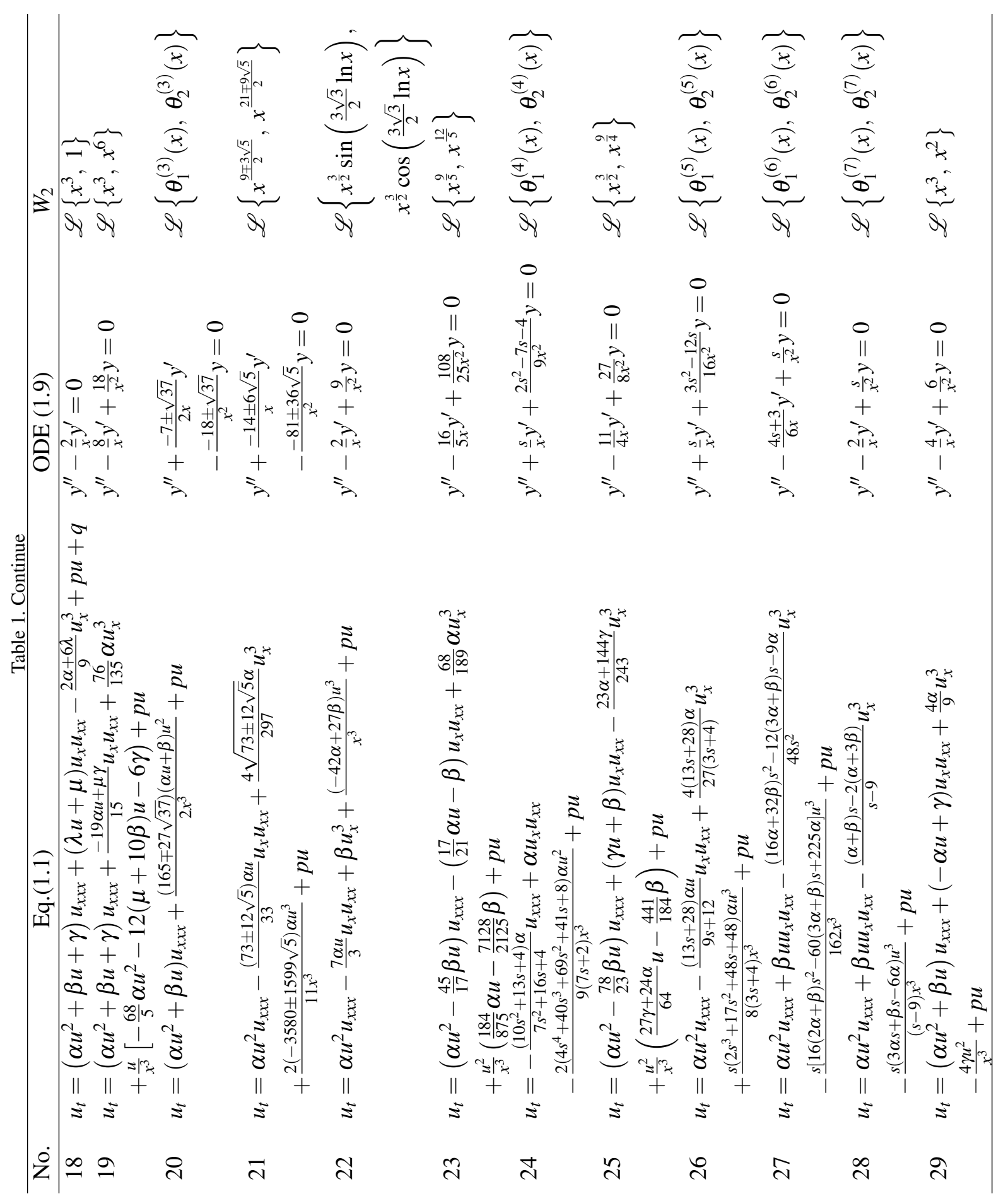




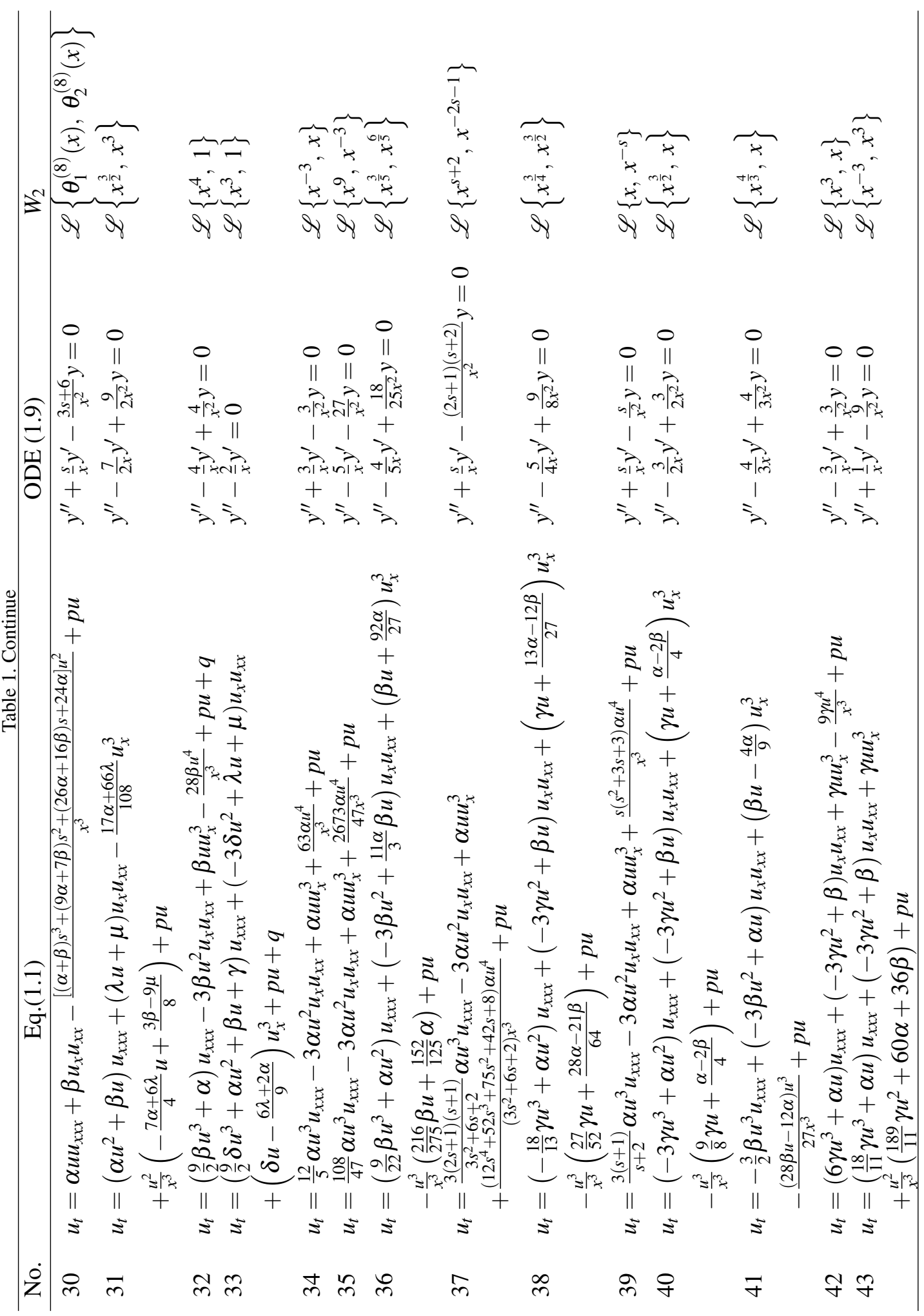




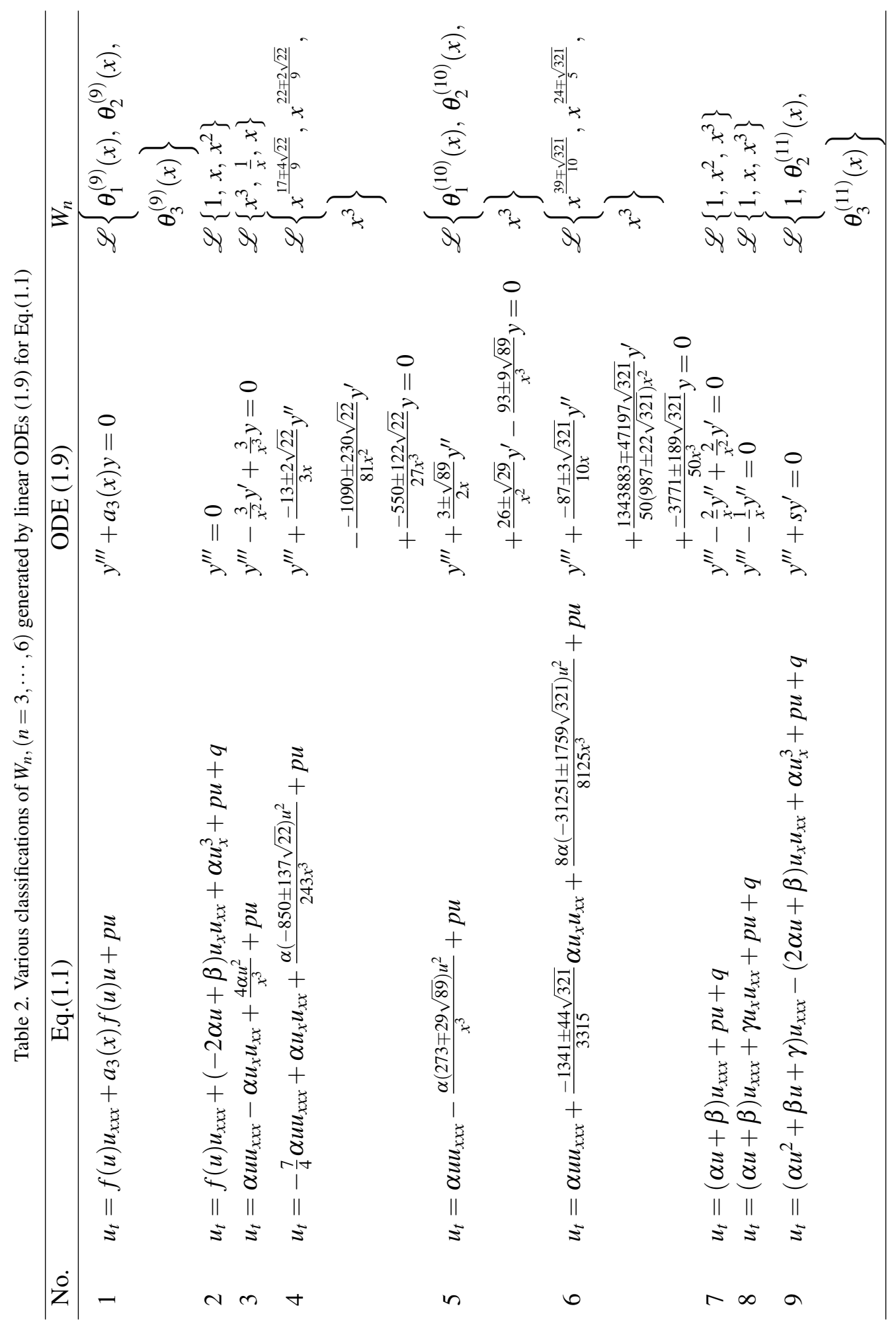




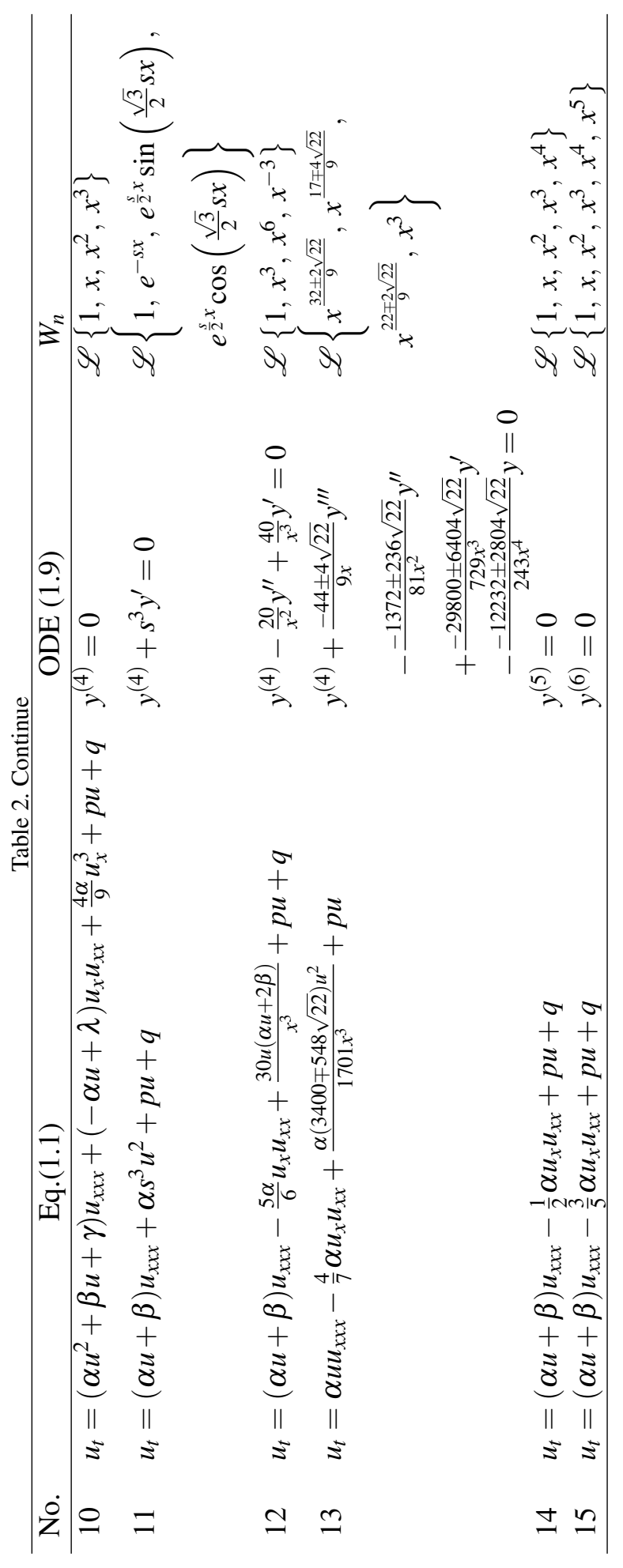




\section{Reductions and exact solutions of concrete examples}

In this section, we present several concrete examples to illustrate our classifications resulted from the invariant subspace method.

Example 1. The equation

$$
u_{t}=\alpha u^{2} u_{x x x}-\frac{(13 s+28) \alpha}{9 s+12} u u_{x} u_{x x}+\frac{4(13 s+28) \alpha}{27(3 s+4)} u_{x}^{3}+\frac{s\left(2 s^{3}+17 s^{2}+48 s+48\right) \alpha}{8(3 s+4) x^{3}} u^{3}+p u
$$

with $s \neq-2,-\frac{4}{3}$ admits the invariant subspace $W_{2}=\mathscr{L}\left\{x^{-\frac{3 s}{4}}, x^{-\frac{s}{4}+1}\right\}$ generated by the linear ODE

$$
y^{\prime \prime}+\frac{s}{x} y^{\prime}+\frac{3 s^{2}-12 s}{16 x^{2}} y=0 .
$$

The corresponding exact solution is given by

$$
u(x, t)=c_{1}(t) x^{-\frac{3 s}{4}}+c_{2}(t) x^{-\frac{s}{4}+1},
$$

where $c_{1} \equiv c_{1}(t)$ and $c_{2} \equiv c_{2}(t)$ satisfy the dynamical system

$$
\begin{aligned}
c_{1}^{\prime} & =\frac{\alpha c_{2}^{3}}{3 s+4}\left(\frac{13}{54} s^{4}+\frac{53}{27} s^{3}+6 s^{2}+\frac{220}{27} s+\frac{112}{27}\right)+p c_{1}, \\
c_{2}^{\prime} & =p c_{2} .
\end{aligned}
$$

When $p=0$, we have

$$
\begin{aligned}
& c_{1}=\frac{\alpha C_{2}^{3} t}{3 s+4}\left(\frac{13}{54} s^{4}+\frac{53}{27} s^{3}+6 s^{2}+\frac{220}{27} s+\frac{112}{27}\right)+C_{1}, \\
& c_{2}=C_{2}
\end{aligned}
$$

and when $p \neq 0$, we have

$$
\begin{aligned}
& c_{1}=\frac{\alpha C_{2}^{3} e^{3 p t}}{2 p(3 s+4)}\left(\frac{13}{54} s^{4}+\frac{53}{27} s^{3}+6 s^{2}+\frac{220}{27} s+\frac{112}{27}\right)+C_{1} e^{p t}, \\
& c_{2}=C_{2} e^{p t}
\end{aligned}
$$

where $C_{1}$ and $C_{2}$ are two arbitrary constants.

Example 2. The equation

$$
u_{t}=-\frac{21 \alpha}{11} u u_{x x x}+\alpha u_{x} u_{x x}-\frac{72 \alpha}{11 x^{3}} u^{2}+p u
$$

admits the invariant subspace $W_{2}=\mathscr{L}\left\{x^{3}, x^{3} \ln x\right\}$ generated by the linear ODE

$$
y^{\prime \prime}-\frac{5}{x} y^{\prime}+\frac{9}{x^{2}} y=0 .
$$

The corresponding exact solution is given by

$$
u(x, t)=c_{1}(t) x^{3}+c_{2}(t) x^{3} \ln x,
$$


where $c_{1} \equiv c_{1}(t)$ and $c_{2} \equiv c_{2}(t)$ satisfy the dynamical system

$$
\begin{aligned}
& c_{1}^{\prime}=5 \alpha c_{2}^{2}+p c_{1}, \\
& c_{2}^{\prime}=p c_{2} .
\end{aligned}
$$

When $p=0$, we have

$$
\begin{aligned}
& c_{1}=5 \alpha C_{2}^{2} t+C_{1}, \\
& c_{2}=C_{2},
\end{aligned}
$$

and when $p \neq 0$, we have

$$
\begin{aligned}
& c_{1}=\frac{5 \alpha C_{2}^{2} e^{2 p t}}{p}+C_{1} e^{p t}, \\
& c_{2}=C_{2} e^{p t}
\end{aligned}
$$

where $C_{1}$ and $C_{2}$ are two arbitrary constants.

Example 3. The equation

$$
u_{t}=\alpha u u_{x x x}-\alpha u_{x} u_{x x}+\frac{4 \alpha u^{2}}{x^{3}}+p u
$$

admits the invariant subspace $W_{3}=\mathscr{L}\left\{x^{3}, \frac{1}{x}, x\right\}$ generated by the linear ODE

$$
y^{\prime \prime \prime}-\frac{3}{x^{2}} y^{\prime}+\frac{3}{x^{3}} y=0 .
$$

The corresponding exact solution is given by

$$
u(x, t)=c_{1}(t) x^{3}+c_{2}(t) \frac{1}{x}+c_{3}(t) x,
$$

where $c_{1}(t), c_{2}(t)$ and $c_{3}(t)$ satisfy the dynamical system

$$
\begin{aligned}
& c_{1}^{\prime}=-8 \alpha c_{1}^{2}+p c_{1}, \\
& c_{2}^{\prime}=8 \alpha c_{1} c_{2}+4 \alpha c_{3}^{2}+p c_{2}, \\
& c_{3}^{\prime}=8 \alpha c_{1} c_{3}+p c_{3} .
\end{aligned}
$$

Example 4. The equation

$$
u_{t}=\left(\alpha u^{2}+\beta u+\gamma\right) u_{x x x}-(2 \alpha u+\beta) u_{x} u_{x x}+\alpha u_{x}^{3}+p u+q
$$

admits the invariant subspace $W_{3}=\mathscr{L}\{1, \sin (\sqrt{s} x), \cos (\sqrt{s} x)\} \quad$ or $\mathscr{L}\{1, \exp (\sqrt{-s} x), \exp (-\sqrt{-s} x)\}$ or $\mathscr{L}\left\{1, x, x^{2}\right\}$ generated by the linear ODE

$$
y^{\prime \prime \prime}+s y=0 .
$$

When $s>0$, the corresponding exact solution has the form

$$
u(x, t)=c_{1}(t)+c_{2}(t) \sin (\sqrt{s} x)+c_{3}(t) \cos (\sqrt{s} x),
$$


where $c_{1}(t), c_{2}(t)$ and $c_{3}(t)$ satisfy the dynamical system

$$
\begin{aligned}
& c_{1}^{\prime}=p c_{1}+q, \\
& c_{2}^{\prime}=s^{\frac{3}{2}} c_{3}\left[\alpha\left(c_{1}^{2}-c_{2}^{2}-c_{3}^{2}\right)+\beta c_{1}+\gamma\right]+p c_{2}, \\
& c_{3}^{\prime}=s^{\frac{3}{2}} c_{2}\left[\alpha\left(-c_{1}^{2}+c_{2}^{2}+c_{3}^{2}\right)-\beta c_{1}-\gamma\right]+p c_{3} .
\end{aligned}
$$

When $s<0$, the corresponding exact solution has the form

$$
u(x, t)=c_{1}(t)+c_{2}(t) \exp (\sqrt{-s} x)+c_{3}(t) \exp (-\sqrt{-s} x),
$$

where $c_{1}(t), c_{2}(t)$ and $c_{3}(t)$ satisfy the dynamical system

$$
\begin{aligned}
& c_{1}^{\prime}=p c_{1}+q, \\
& c_{2}^{\prime}=(-s)^{\frac{3}{2}} c_{2}\left[\alpha\left(c_{1}^{2}-4 c_{2} c_{3}\right)+\beta c_{1}+\gamma\right]+p c_{2}, \\
& c_{3}^{\prime}=(-s)^{\frac{3}{2}} c_{3}\left[\alpha\left(-c_{1}^{2}+4 c_{2} c_{3}\right)-\beta c_{1}-\gamma\right]+p c_{3} .
\end{aligned}
$$

When $s=0$, the corresponding exact solution has the form

$$
u(x, t)=c_{1}(t)+c_{2}(t) x+c_{3}(t) x^{2},
$$

where $c_{1}(t), c_{2}(t)$ and $c_{3}(t)$ satisfy the dynamical system

$$
\begin{aligned}
& c_{1}^{\prime}=-2 \beta c_{2} c_{3}-4 \alpha c_{1} c_{2} c_{3}+\alpha c_{2}^{3}+p c_{1}+q, \\
& c_{2}^{\prime}=-4 \beta c_{3}^{2}-8 \alpha c_{1} c_{3}^{2}+2 \alpha c_{2}^{2} c_{3}+p c_{2}, \\
& c_{3}^{\prime}=p c_{3} .
\end{aligned}
$$

Example 5. The equation

$$
u_{t}=(\alpha u+\beta) u_{x x x}-\frac{5 \alpha}{6} u_{x} u_{x x}+\frac{30 u(\alpha u+2 \beta)}{x^{3}}+p u+q
$$

admits the invariant subspace $W_{4}=\mathscr{L}\left\{1, x^{3}, x^{6}, x^{-3}\right\}$ generated by the linear ODE

$$
y^{(4)}-\frac{20}{x^{2}} y^{\prime \prime}=0 .
$$

The corresponding exact solution has the form

$$
u(x, t)=c_{1}(t)+c_{2}(t) x^{3}+c_{3}(t) x^{6}+c_{4}(t) x^{-3},
$$

where $c_{i} \equiv c_{i}(t),(i=1, \cdots, 4)$ satisfy the dynamical system

$$
\begin{aligned}
& c_{1}^{\prime}=66 \alpha c_{1} c_{2}+135 \alpha c_{3} c_{4}+66 \beta c_{2}+p c_{1}+q, \\
& c_{2}^{\prime}=21 \alpha c_{2}^{2}+180 \alpha c_{1} c_{3}+180 \beta c_{3}+p c_{2}, \\
& c_{3}^{\prime}=81 \alpha c_{2} c_{3}+p c_{3} \\
& c_{4}^{\prime}=30 \alpha c_{1}^{2}-9 \alpha c_{2} c_{4}+60 \beta c_{1}+p c_{4} .
\end{aligned}
$$

Example 6. The equation

$$
u_{t}=(\alpha u+\beta) u_{x x x}-\frac{3}{5} \alpha u_{x} u_{x x}+p u+q
$$


admits the invariant subspace $W_{6}=\mathscr{L}\left\{1, x, x^{2}, x^{3}, x^{4}, x^{5}\right\}$ generated by the linear ODE

$$
y^{(6)}=0
$$

The corresponding exact solution has the form

$$
u(x, t)=c_{1}(t)+c_{2}(t) x+c_{3}(t) x^{2}+c_{4}(t) x^{3}+c_{5}(t) x^{4}+c_{6}(t) x^{5},
$$

where $c_{i} \equiv c_{i}(t),(i=1, \cdots, 6)$ satisfy the dynamical system

$$
\begin{aligned}
c_{1}^{\prime} & =-\frac{6}{5} \alpha c_{2} c_{3}+6 \alpha c_{1} c_{4}+6 \beta c_{4}+p c_{1}+q, \\
c_{2}^{\prime} & =-\frac{12}{5} \alpha c_{3}^{2}+24 \alpha c_{1} c_{5}+\frac{12}{5} \alpha c_{2} c_{4}+24 \beta c_{5}+p c_{2}, \\
c_{3}^{\prime} & =-\frac{24}{5} \alpha c_{3} c_{4}+60 \alpha c_{1} c_{6}+\frac{84}{5} \alpha c_{2} c_{5}+60 \beta c_{6}+p c_{3}, \\
c_{4}^{\prime} & =-\frac{24}{5} \alpha c_{4}^{2}+48 \alpha c_{2} c_{6}+\frac{24}{5} \alpha c_{3} c_{5}+p c_{4}, \\
c_{5}^{\prime} & =-6 \alpha c_{4} c_{5}+30 \alpha c_{3} c_{6}+p c_{5}, \\
c_{6}^{\prime} & =-\frac{24}{5} \alpha c_{5}^{2}+12 \alpha c_{4} c_{6}+p c_{6} .
\end{aligned}
$$

\section{Conclusions and discussions}

In this paper, we have applied the invariant subspace method to the study of exact solutions to Eq.(1.1). A class of evolution equations admitting invariant subspaces $W_{n} \equiv \mathscr{L}\left\{f_{1}(x), \cdots, f_{n}(x)\right\}$ generated by linear ODEs (1.9) of order $n$ less than seven are computed and listed in Table 1 and Table 2. The resulting exact solutions of the corresponding equations have the forms of generalized separation of variables $u=\sum_{i=1}^{n} c_{i}(t) f_{i}(x)$ where the coefficient functions $c_{i}(t),(i=1, \cdots, n)$ satisfy $n$-dimensional dynamical systems. Generally speaking, such exact solutions can not be obtained within the frameworks of the Lie point symmetry method and the nonclassical symmetry method.

Although the invariant subspace method is effective, we would like to point out that the refined invariant subspace method produces wider invariant subspaces of exact solutions defined by finitedimensional dynamical systems [9]. There are also many other interesting problems of applying the invariant subspace method method or the refine invariant subspace method. For example, how to determine higher-order NLEEs and systems of higher-order NLEEs? How to deal with the initial value problems? How to present resonant solutions with generalized separation of variables for generalized bilinear and trilinear differential equations [12-14]?

\section{Acknowledgments}

We are appreciated to the referee and editor for their constructive suggestions and helpful comments. This work is in part supported by the National Natural Science Foundations of China (Grant Nos. 11371323, 11371326 and 11271008), Zhejiang Innovation Project of China (Grant No. T200905), and the First-class Discipline of Universities in Shanghai and the Shanghai Univ. Leading Academic Discipline Project (No. A.13-0101-12-004). 
Invariant subspaces of third-order nonlinear evolution equations

\section{References}

[1] A.S. Fokas, Q.M. Liu, Nonlinear interaction of travelling waves of nonintegrable equations, Phys. Rev. Lett. 72 (1994) 3293-3296.

[2] V.A. Galaktionov, S.R. Svirshchevskii, Exact solutions and invarinat subspaces of nonlinear partial differential equations in mechanics and physics, Appl. math. nonl. sci. series $\mathbf{1 0}$ (Chapman and Hall/CRC, London, 2007).

[3] R.K. Gazizov, A.A. Kasatkin, Construction of exact solutions for fractional order differential equations by the invariant subspace method, Comput. Math. Appl. 66 (2013) 576-584.

[4] D. Gómez-Ullate, N. Kamran, R. Milson, Structure theorems for linear and non-linear differential operators admitting invariant polynomial subspaces, Discrete Contin. Dyn. Syst. 18 (2007) 85-106.

[5] N. Kamran, R. Milson, P.J. Olver, Invariant modules and the reduction of nonlinear partial differential equations to dynamical systems, Adv. Math. 156 (2000) 286-319.

[6] O.V. Kaptsov, I.V. Verevkin, Differential constraints and exact solutions of nonlinear diffusion equations, J. Phys. A: Math. Gen. 36 (2003) 1401-1414.

[7] J.R. King, Exact polynomial solutions to some nonlinear diffusion equations, Physica D 64 (1993) $35-65$.

[8] Y.Z. Lin, Y.P. Liu, Z.B. Li, Exact solutions for pattern formation in a reaction-diffusion system, Int. J. Nonlinear Sci. Numer. Simul. 14 (2013) 307-315.

[9] W.X. Ma, A refined invariant subspace method and applications to evolution equations, Sci. China Math. 55 (2012) 1769-1778.

[10] W.X. Ma, Y.P. Liu, Invariant subspaces and exact solutions of a class of dispersive evolution equations, Commun. Nonlinear Sci. Numer. Simulat. 17 (2012) 3795-3801.

[11] W.X. Ma, An extended Harry Dym hierarchy, J. Phys. A: Math. Theor. 43 (2010) 165202 (13pp).

[12] W. X. Ma, Generalized bilinear differential equations, Stud. Nonlinear Sci. 2 (2011) 140-144.

[13] W.X. Ma, Bilinear equations and resonant solutions characterized by Bell polynomials, Rep. Math. Phys. 72 (2013) 41-56.

[14] W.X. Ma, Trilinear equations, Bell polynomials, and resonant solutions, Front. Math. China 8 (2013) 1139-1156.

[15] C.Z. Qu, Group classification and generalized conditional symmetry reduction of the nonlinear diffusion-convection equation with a nonlinear source, Stud. Appl. Math. 99 (1997) 107-136.

[16] C.Z. Qu, C.R. Zhu, Classification of coupled systems with two-component nonlinear diffusion equations by the invrainat subspace method, J. Phys. A: Math. Theor. 42 (2009) 475201 (27pp).

[17] S.F. Shen, C.Z. Qu, Y.Y. Jin, L.N. Ji, Maximal dimension of invariant subspaces to systems of nonlinear evolution equations, Chin. Annal. Math. Series B 33 (2012) 161-178.

[18] J.Q. Song, S.F. Shen, Y.Y. Jin, J. Zhang, New maximal dimension of invariant subspaces to coupled systems with two-component equations, Commun. Nonlinear Sci. Numer. Simulat. 18 (2013) 29842992.

[19] S. Svirshchevskii, Ordinary differential operators possessing invariant subspaces of polynomial type, Commun. Nonlinear Sci. Numer. Simulat. 9 (2004), 105-115.

[20] R.Z. Zhdanov, Conditional Lie-Bäcklund symmetry and reductions of evolution equations, J. Phys. A: Math. Gen. 28 (1995) 3841-3850.

[21] C.R. Zhu, C.Z. Qu, Maximal dimension of invariant subspaces admitted by nonlinear vector differential operators, J. Math. Phys. 52 (2011) 043507 (15pp). 